\title{
Methodology to Promote Physical Activity Monitoring Adherence in Youth with Autism Spectrum Disorder
}

\author{
Janet L. Hauck ${ }^{1 *}$, Leah R. Ketcheson ${ }^{2 \dagger}$ and Dale A. Ulrich ${ }^{2}$ \\ 'Physical Activity for Youth with Disabilities (PLAY'D) Laboratory, Department of Kinesiology, Michigan State University, East \\ Lansing, MI, USA, ${ }^{2}$ Center for Physical Activity and Health in Pediatric Disabilities, School of Kinesiology, University of \\ Michigan, Ann Arbor, MI, USA
}

\section{OPEN ACCESS}

Edited by:

Samuel W. Logan,

Oregon State University, USA

Reviewed by:

Kumaravel Rajakumar,

University of Pittsburgh

School of Medicine, USA

Linda Mullin Elkins,

Life University, USA

Suzanna R. Dillon,

Texas Woman's University, USA

${ }^{*}$ Correspondence:

Janet L. Hauck

hauckja1@msu.edu

${ }^{+}$Present address:

Leah R. Ketcheson,

Kinesiology, Health and Sport Studies, Wayne State University,

Detroit, MI, USA

Specialty section: This article was submitted to Child Health and Human Development,

a section of the journal

Frontiers in Public Health

Received: 29 April 2016 Accepted: 08 September 2016 Published: 27 September 2016

Citation:

Hauck JL, Ketcheson LR and Ulrich DA (2016) Methodology to Promote Physical Activity Monitoring

Adherence in Youth with Autism

Spectrum Disorder.

Front. Public Health 4:206.

doi: 10.3389/fpubh.2016.00206
Background: Objective physical activity (PA) monitoring via accelerometry is both costly and time consuming. Furthermore, overall adherence to a monitoring protocol is often complicated by disability. Therefore, it is essential that strategies for supporting accelerometer wear for youth with disabilities are maximized. The purpose of this perspective was to provide researchers a set of efficacious PA monitoring strategies based on the retrospective examination of support methodology on adherence rates for youth with autism spectrum disorder (ASD).

Method: Accelerometer data were collected from 163 participants with ASD in three independent cohorts. Each cohort was provided a varying set of support strategies to help maximize adherence. Chi-square analysis was used to determine differences in adherence between each cohort.

Results: Adherence rates significantly increased from $51.9 \%$ in cohort 1 to $88.7 \%$ in cohort $2\left[\chi^{2}(1)=18.333, p<0.001\right]$ and again from $88.7 \%$ in cohort 2 to $97.4 \%$ in cohort $3\left[\chi^{2}(1)=2.663, p=0.103\right]$. The greatest increase in adherence was observed from $51.9 \%$ in cohort 1 to $97.4 \%$ in cohort $3\left[\chi^{2}(1)=19.837, p<0.001\right]$. Support strategies associated with these increases included (1) social story, (2) incentive, (3) concealing techniques, and (4) $24 \mathrm{~h} /$ day wear instructions.

Conclusion: Adherence to PA measurement increased when additional support strategies were utilized in combination with a traditional protocol. We recommend these support methodology to be considered as preliminary best practices when measuring objective PA in youth with ASD with likely success in other disability populations.

Keywords: accelerometer, measurement, pediatrics, disabilities, pedometer, exercise, obesity, adapted

\section{INTRODUCTION}

Autism spectrum disorder (ASD) is the fastest growing developmental disability in the United States (1); as such, there is a critical need to research and intervene on the modifiable factors contributing to known health disparities, such as rising rates of overweight and obesity $(2-4)$. The prevalence of obesity in children with ASD aged 2-19 years is estimated at over $30 \%$ (4). One factor which has surfaced 
as a major health concern contributing to the obesity epidemic is the rise in physical inactivity (5). Evidence suggests that both typically developing children and children with disabilities are falling well below recommended physical activity (PA) guidelines with increasing sedentary behavior as they age $(2,6-10)$. In addition, given the current spot light on motor skill competence in children with ASD, motor skill interventions focused on motor and PA outcomes are gaining significant attention yielding the need for improved measurement methodology $(8,11-20)$. In order to maintain insight of the levels and patterns of PA in youth, valid and reliable methods of objective PA measurement must be incorporated. Therefore, it is imperative to continue to evolve evidence-based best practices in activity measurement to enhance research efforts in understanding and addressing this global epidemic.

Current best practices in activity measurement include an objective measurement of PA using motion detectors such as an accelerometer which is worn around the waist, wrist, or ankle (21). Despite recent reports highlighting the importance of measuring PA using an objective method (21), many researchers still resort to implement self-reported and recall instruments. Common methods include retrospective measurement of previous activity with recall varying from $24 \mathrm{~h}$ to 1 year, a parental report of the child's activity, or an activity interview with a log (22). There are several issues with self-reported measures, including inaccurate representation on the frequency, duration, and intensity of PA following bouts of activity which are retrospectively recalled (22). Additionally, youth and their caregivers have a tendency to over-report their PA (21). Despite these issues, self-reported questionnaires are often considered for cost efficiency reasons. Caution, however, should be taken when considering results from large epidemiological studies, with outcomes associated with programing or intervention recommendations when recall methodology is used. To avoid misrepresenting the levels of PA in youth, particularly those most sensitive to program modifications (children with disabilities), employing an objective measurement such as accelerometry, can more accurately inform policy makers and service providers regarding the current PA behavior of targeted populations.

An important component used to inform PA measurement methodology for future research should be the reporting of adherence results from prior studies. Unfortunately, reporting of adherence to PA measurement procedures is rarely published $(7,23,24)$. Adherence to accelerometer measurement procedures is likely to vary by population (children or adults) and experience (no. of observations). However, reporting the number of participants who are able to successfully achieve the minimum amount of wear needed to meet reliability criteria provides an accurate estimate of participants who may potentially adhere in future studies; valuable information which can be used to inform sample size and research budgets. Similarly, studies examining the minimum days and hours of PA monitoring required for reliability have the potential to reduce participant burden while increasing adherence $(25,26)$. A recent study examined the minimum number of days required to reliably measure PA in youth with developmental disabilities (27). Results indicated that 4,6 , and 8 days of monitoring were required to reliably quantify typical levels of PA during the week, weekend, and combined week and weekend, respectively (27). Additional studies examining the minimum number of days required for monitoring will provide further support for these findings, and ultimately provide valuable information which can be used to develop best practices when examining PA in youth with disabilities.

Another consideration in the measurement of objective PA via accelerometry in youth is cost. The majority of expenses are typically associated with cost of the device, which can range from $\$ 250$ to $\$ 450$. Required software costs also vary substantially. In addition to hardware and software costs, expenses relating to research procedures can accumulate. One such cost is providing incentive. Since PA monitoring can be burdensome for some children, particularly those with disabilities, it may be wise to provide an incentive for wearing the monitor; a recommendation that is substantiated by the current report. Also, the cost of postage should be considered given that monitors are most efficiently delivered and returned via mail. Finally, data reduction and analysis are time consuming; therefore, it is also wise to consider the cost of hiring personnel to assist with this process. Despite these considerable expenses which are further burdened by poor adherence, the reporting of strategies to increase PA monitoring adherence is scarce.

Taken together, objective PA monitoring via accelerometry is both costly and time consuming. Therefore, it is essential that strategies for supporting accelerometer wear for youth with disabilities are maximized (24). The purpose of this retrospective investigation was to compare adherence rates of accelerometer measurement protocols across three independent cohorts, to differentiate the effects of support strategies offered to increase protocol adherence, and to offer our perspective on accelerometer adherence promoting strategies that should be considered when monitoring PA in children and youth ASD.

\section{METHOD}

\section{Participants}

We performed a retrospective comparison of PA monitoring adherence data obtained from three independent cohorts (28-30). For each cohort, an independent sample was recruited to participate in an intervention which included the measurement of habitual PA via an accelerometer. A total of 163 youth aged 9-18 years with ASD participated (cohort 1, $n=27$; cohort 2, $n=97$; cohort 3, $n=39$ ). Demographic data for each cohort are summarized in Table 1. The research protocol was approved by the Health Sciences and Behavioral Sciences Institutional Review Board. All participants were consented to participate and assent was obtained from each.

\section{Procedure}

A baseline measure of objective PA via an accelerometer was collected for each cohort. Type of accelerometer used was individually decided by the research team for each cohort and therefore varies. The Actical accelerometer (Actical, Philips Respironics; Bend, OR, USA) was used for cohorts 1 and 2. The Actigraph accelerometer (Actigraph GT3X, Actigraph; Pensacola, FL, USA) was used for cohort 3. These devices are very similar in size and shape and therefore are not considered as a factor affecting adherence. 


\section{Data Reduction}

Data were reduced using specific reliability criteria and validated cut points $(31,32)$. Data were considered reliable when the accelerometer was worn for a minimum of $10 \mathrm{~h}$ on 4 days in short succession, one of which must have been a weekend day. Data were only included in further analysis if reliability criteria were met. We used reliability criteria as the critical factor in determining whether adherence was met. If criteria were met, then adherence to the PA protocol was met, yielding usable and reliable data. If criteria were not met, then adherence was also not met.

\section{Adherence Methodology}

For each cohort, a standardized set of adherence strategies were provided to participants in an effort to increase the likelihood that reliability criteria were met. With each successive cohort,

TABLE 1 | Demographic data of participants by cohort.

\begin{tabular}{lccc}
\hline Characteristics & $\begin{array}{c}\text { Cohort 1 } \\
\boldsymbol{n}=\mathbf{2 7}\end{array}$ & $\begin{array}{c}\text { Cohort 2 } \\
\boldsymbol{n}=\mathbf{9 7}\end{array}$ & $\begin{array}{c}\text { Cohort 3 } \\
\boldsymbol{n}=\mathbf{3 9}\end{array}$ \\
\hline \% of female & 27 & 23 & 19 \\
Age (years) & $11.9 \pm 2.4$ & $12.1 \pm 2.3$ & $11.8 \pm 2.5$ \\
Height (cm) & $147.0 \pm 12.9$ & $149.6 \pm 13.3$ & $145.1 \pm 10.9$ \\
Weight (kg) & $46.9 \pm 19.3$ & $48.8 \pm 19.9$ & $40.4 \pm 14.3$ \\
BMl & $20.6 \pm 7.0$ & $21.1 \pm 5.4$ & $18.8 \pm 4.3$ \\
SRS score & - & $81.0 \pm 13.9$ & $81.8 \pm 13.0$ \\
ADOS-2 CSS & - & - & $6.75 \pm 1.5$ \\
WASI-2 & - & $81.0 \pm 18.9$ & $85.5 \pm 19.7$ \\
PPVT-4 & $74.9 \pm 26.4$ & - & -
\end{tabular}

BMI, Body Mass Index; SRS, Social Responsiveness Scale; ADOS, Autism Diagnostic Observation Schedule; CSS, Calibrated Severity Score; WASI, Wechsler Abbreviated

Scale of Intelligence; PPVT, Peabody Picture Vocabulary Test. new adherence strategies were added to the original protocol procedures at the discretion of the research team. Original protocol procedures used consistently for all three cohorts included a 1-week monitoring period, providing written instructions, a monitoring log, and providing a self-addressed and stamped envelope for returning the monitor. Additional adherence strategies implemented for cohorts 2 and 3 included verbal instructions, in-person training, providing a social story, increased daily wear instructions to $24 \mathrm{~h} /$ day, concealing and decorating wear techniques, and incentive payment (cohort 3 only). Due to the post hoc nature of this investigation, we were unable to manipulate support strategies among the three samples in a systematic way, limiting specific interpretations. Please see Table 2 for a full description of protocol procedures and adherence strategies as well as which cohorts incorporated their use.

\section{Statistical Analysis}

All PA data were reduced with computer software using the previously mentioned reliability criteria. Actical data were reduced using a specially designed computer program (33). Actigraph data were reduced using ActiLife 6.0. Adherence was used as a binary outcome variable and classification of "adherence" or "non-adherence" was based on whether or not reliability criteria were met $(10 \mathrm{~h} / 4$ days +1 weekend day). Analyses included determining the percentage of the sample that adhered to the PA measurement protocol for each study. Chi-square analysis was used to determine differences in adherence between each cohort. Support strategies utilized were then compared and contrasted accounting for observed differences in adherence outcomes.

All statistical analyses were conducted using IBM SPSS Statistics 20 (SPSS Inc., Chicago, IL, USA). Differences were considered significant at an alpha level of 0.05 .

TABLE 2 | Use of traditional accelerometry procedures and additional support strategies by cohort.

\begin{tabular}{|c|c|c|c|c|}
\hline Adherence supports $^{a}$ & Brief description & $\begin{array}{c}\text { Cohort } 1 \\
n=27\end{array}$ & $\begin{array}{c}\text { Cohort } 2 \\
n=97\end{array}$ & $\begin{array}{c}\text { Cohort } 3 \\
n=39\end{array}$ \\
\hline Monitoring period & Amount of time participants are instructed to wear accelerometer & 1 week & 1 week & 1 week \\
\hline \multicolumn{5}{|l|}{ Method of delivery } \\
\hline Mail & Participants received accelerometer by mail & $x$ & & $x$ \\
\hline In person & Participants received accelerometer in person & & $x$ & \\
\hline \multicolumn{5}{|l|}{ Instructions } \\
\hline Written ${ }^{b}$ & Provided a simple letter detailing instructions & $x$ & $x$ & $x$ \\
\hline Verbal & Provided in-person verbal instructions & & $x$ & \\
\hline In-person training & Conducted home visits to provide in-person training & & $\mathrm{x}$ & \\
\hline Social story & Provided social story to increase comprehension and reduce anxiety & & $\mathrm{x}$ & $x$ \\
\hline Monitor log & Provided a monitoring log to record non-wear periods & $x$ & $x$ & $x$ \\
\hline \multicolumn{5}{|l|}{ Wear time instructions } \\
\hline Waking hours & Participants instructed to wear accelerometer during waking hours & $x$ & & \\
\hline 24 h/day & Participants instructed to wear accelerometer $24 \mathrm{~h} /$ day & & $x$ & $x$ \\
\hline \multicolumn{5}{|l|}{ Wear techniques } \\
\hline Concealing & Taught participants to conceal accelerometer under clothing & & $\mathrm{x}$ & $x$ \\
\hline Decorating & Allowed participants to decorate accelerometer with stickers & & $x$ & \\
\hline Returned by mail ${ }^{b}$ & Participants returned accelerometer in pre addressed/stamped envelop & $x$ & $x$ & $x$ \\
\hline Incentive & Incentive provided upon return of accelerometer with complete data & & & $x$ \\
\hline
\end{tabular}

$x=$ used adherence support.

a Supports are presented as a semi-sequential timeline of when supports are typically utilized during physical activity measurement.

${ }^{b}$ Original protocol procedures. 


\section{RESULTS}

The results present post hoc comparison of accelerometry adherence rates from three independent cohorts, each of which incorporated a varying combination of support methodology. For cohort 1, 14 of 27 participants met reliability criteria indicating $51.9 \%$ adherence. For cohort 2, 86 of 97 participants met reliability criteria indicating $88.7 \%$ adherence. For cohort 3,38 of 39 participants met reliability criteria indicating $97.4 \%$ adherence.

To evaluate the meaningfulness of additional adherence support strategies from one cohort to another, group differences of adherence were calculated using chi-square. Adherence significantly increased between cohorts 1 and $2\left[\chi^{2}(1)=18.333\right.$, $p<0.001]$. Additional supports included (1) in-person delivery of accelerometer, (2) verbal instructions, (3) in-person training, (4) providing a social story, (5) increased daily wear instructions to $24 \mathrm{~h} /$ day, (6) concealing wear techniques, and (7) decorating wear techniques.

There was a non-significant increase in adherence between cohorts 2 and $3\left[\chi^{2}(1)=2.663, p=0.103\right]$. One additional support was included during that period. Incentive payment was provided to participants upon the return of the accelerometer containing complete data. Supports that were omitted for cohort 3 which had been previously utilized for cohort 2 included (1) in-person delivery of accelerometer, (2) verbal instructions, (3) in-person training, and (4) decorating wear techniques.

Adherence significantly increased between cohorts 1 and 3 $\left[\chi^{2}(1)=19.837, p<0.001\right]$. Additional supports included (1) providing a social story, (2) increased daily wear instructions to $24 \mathrm{~h} /$ day, (3) concealing wear techniques, and (4) providing incentive payment.

\section{DISCUSSION}

Successful adherence to objective PA measurement protocol for youth with ASD aged 9-18 years was increased over the progression of three independent cohorts. This improvement coincides with the addition of many adherence support strategies. The contribution of each subsequent support is suspected of influencing the significant increase observed in adherence. These results are positive and the first to contribute to the development of best practices when conducting objective PA measurement via an accelerometer in youth with disabilities.

Adherence rates significantly increased by $45.5 \%$ from cohort 1 to cohort 3. Adherence support strategies that both cohorts 2 and 3 shared in common include providing a social story, increasing wear time to $24 \mathrm{~h} /$ day, and offering concealing tips to participants. These supports were not included in the first cohort. Given the progression of increased adherence, it is likely that the addition of these supports to the PA measurement protocol influenced adherence. However, caution must be warned in making an assumption of causation given the lack of a true control group.

To further facilitate improved adherence, one additional support was utilized for cohort 3 which had not been previously attempted. Participants were offered a small monetary incentive if they returned the accelerometer with complete data, meaning that they wore the monitor long enough to accumulate the minimum amount of data to achieve reliability. The use of the incentive support stimulated an additional non-significant increase in adherence of $8.7 \%$ between cohorts 2 and 3, even in the absence of other helpful supports utilized for cohort 2. Anecdotal evidence suggested that providing the incentive gave participants (or their caregivers) a perceived benefit in completing the PA measurement, thereby increasing dedication to the task. In addition, the research garnished a higher return rate of monitors when incentive was offered.

We believe that the incentive support greatly improved adherence, to the extent that we are now tentative in recommending other supports that were only offered to cohort 2 . Those supports included in-person delivery of the accelerometer with in-person training and verbal instructions. These supports clearly increased adherence by $36.8 \%$ between cohorts 1 and 2 ; however, we demonstrated that adherence continued to improve even after omitting these supports for cohort 3 when an incentive is offered. Given the increased costs to the research in time, effort, and budget, we do not recommend in-person delivery, in-person training, or verbal instructions be incorporated into your measurement protocol unless resources allow for these extra costs, so long as incentive is offered following the return of the accelerometer with complete data.

Limitations exist which could affect interpretations. First and foremost, we are not examining the effects of sample characteristics to adherence of accelerometer procedures, but rather the effects of the adherence supports and instructional procedures provided by the research team. Assessments utilized to summarize IQ and ASD severity varied between cohorts, essentially limiting such analyses. Despite this, IQ determined via the Peabody Picture Vocabulary Test- 4 and the Wechsler Abbreviated Scale of Intelligence-2 resulted in comparable IQs for all cohorts. Minimal differences in BMI are also noted, with no statistically significant differences between cohorts. Despite this, the relation of adherence rates to BMI was not investigated. Additionally, we are not comparing PA between the samples. For that reason, information regarding epoch length, cut points, and PA intensities are not reported. Finally, this retrospective data analysis was performed after the conclusion of measurement of all cohorts, yielding us little control over the systematic planning of support use, accelerometer type, or population. Having said this, the research made every effort to control for environmental and participant demographics. Measurement occurred during a 1-week period in the spring time in the same geographical region in the United States during each study. The sample included only youth diagnosed with ASD. The mean age of participants across studies was stable, only varying by a few months. Accelerometer model varied, but most would conclude this had little impact on adherence given the size similarities between the Actical and Actigraph.

These limitations prompt caution regarding causation. Given the lack of a true control group or the inability to manipulate support use in a systematic way, it is difficult to quantify the individual effects of each strategy. Also, we are unable to differentiate between child and parent driven adherence but can attest that all supports were provided to parents by the research team and in many cases were disseminated to the child from the parent. 
Finally, we acknowledge our limited ability to remark on the influence of autism severity and functional status on adherence rates. Given inherent variability in the severity and functional status of children on the autism spectrum, a well-controlled prospective study is warranted to validate the effectiveness of these strategies for enhancing PA monitoring adherence in children with comparable functioning.

As a result of incorporating multiple adherence supports into the practice of objective PA measurement via an accelerometer for youth with ASD, adherence rates increased yielding more reliable data and fewer missing data. One common issue experienced by PA researchers are participants who wear the device as described but fall just short of reaching the minimum amount of wear time needed to meet reliability criteria. Oftentimes, these participants' miss reliability criteria by only a few hours on a given day or by missing a weekend day of wear. For this reason, it is important that research continue to examine the minimum wear criteria needed to produce reliable data in special populations. If criteria are reduced, adherence will likely be increased. A reduction of wear time would decrease research costs and ease burden for participants.

In conclusion, adherence to objective PA measurement via an accelerometer can be increased when extra support strategies are utilized. We feel that adherence is stimulated most by increasing wear time to $24 \mathrm{~h}$ /day, providing a social story, offering concealing tips to participants, and providing an incentive upon the return of the accelerometer with complete data. These support strategies should be used in combination with traditional protocol components including a 1-week monitoring period,

\section{REFERENCES}

1. Baio J. Prevalence of Autism Spectrum Disorders - Autism and Developmental Disabilities Monitoring Network, 14 Sites, United States, 2008. Atlanta, GA: Centers for Disease Control and Prevention (2012) Vol. 61, 1-19. Morbidity and Mortality Weekly Report. Surveillance Summaries.

2. McCoy SM, Jakicic JM, Gibbs BB. Comparison of obesity, physical activity, and sedentary behaviors between adolescents with autism spectrum disorders and without. J Autism Dev Disord (2016) 46(7):2317-26. doi:10.1007/ s10803-016-2762-0

3. Egan AM, Dreyer ML, Odar CC, Beckwith M, Garrison CB. Obesity in young children with autism spectrum disorders: prevalence and associated factors. Childhood Obes (2013) 9(2):125-31. doi:10.1089/chi.2012.0028

4. Curtin C, Anderson SE, Must A, Bandini L. The prevalence of obesity in children with autism: a secondary data analysis using nationally representative data from the National Survey of Children's Health. BMC Pediatr (2010) 10(1):11. doi:10.1186/1471-2431-10-11

5. Physical Activity Guidelines Advisory Committee. Physical Activity Guidelines Advisory Committee Report, 2008. Washington, DC: US Department of Health and Human Services (2008). p. A1-14.

6. Tyler K, MacDonald M, Menear K. Physical activity and physical fitness of school-aged children and youth with autism spectrum disorders. Autism Res Treatment (2014) 2014:312163. doi:10.1155/2014/312163

7. Esposito PE, MacDonald M, Hornyak JE, Ulrich DA. Physical activity patterns of youth with down syndrome. Intellect Dev Disabil (2012) 50(2):109-19. doi:10.1352/1934-9556-50.2.109

8. MacDonald M, Esposito P, Ulrich D. The physical activity patterns of children with autism. BMC Res Notes (2011) 4(1):422. doi:10.1186/1756-0500-4-422

9. Kim S-Y. Assessing physical activity levels of students with disabilities in physical education? J Phys Educ Recreat Dance (2009) 80(3):3-5. doi:10.1080/ 07303084.2009.10598286 providing written instructions, a monitoring log, and providing a self-addressed stamped return envelope. These methods were recently utilized with a younger sample of children with ASD with a $95 \%$ adherence rate $(11,34)$. This suggests utility of these supports in younger samples. These strategies can be generalized to objective PA monitoring using other devices such as pedometers and for use in children without disabilities. Adding these strategies to your pediatric PA measurement protocol is likely to increase research efficiency and decrease non-incentive related costs. Finally, we advise these support methodology be strongly considered as preliminary best practices when measuring objective PA in youth with ASD.

\section{AUTHOR CONTRIBUTIONS}

$\mathrm{JH}$ contributed to the research design, data collection, statistical analysis, and preparation of the draft of the manuscript. LK contributed to data collection, statistical analysis, and editing the draft of the manuscript. DU contributed to the research design, securing funds, data collection, and editing the draft of the manuscript.

\section{FUNDING}

This research study was funded in part by the National Institute for Disability Research and Rehabilitation (NIDRR; H133G090006) and the U.S. Office of Special Education Programs (OSEP; H325D020028).

10. Tucker P. The physical activity levels of preschool-aged children: a systematic review. Early Child Res Q (2008) 23(4):547-58. doi:10.1016/ j.ecresq.2008.08.005

11. Ketcheson L, Hauck J, Ulrich D. The effects of an early motor skill intervention on motor skills, levels of physical activity and socialization in young children with Autism Spectrum Disorder: a pilot study. Autism (2016). doi:10.1177/1362361316650611

12. Bremer E, Lloyd M. School-based fundamental-motor-skill intervention for children with autism-like characteristics: an exploratory study. Adapted Phys Activity Q (2016) 33(1):66-88. doi:10.1123/APAQ.2015-0009

13. Bremer E, Balogh R, Lloyd M. Effectiveness of a fundamental motor skill intervention for 4-year-old children with autism spectrum disorder: a pilot study. Autism (2015) 19(8):980-91. doi:10.1177/1362361314557548

14. Hauck JL, Jeong I, Esposito P, MacDonald M, Ulrich DA. Benefits of bicycle riding in adolescents with autism and Down syndrome. Res Q Exerc Sport (2014) 85(S1):68.

15. Pitchford EA, Hauck JL, Ketcheson LR, Reynolds JL, Ulrich DA. Use it or lose it: bicycle riding maintenance in autism. Res Q Exerc Sport (2014) 85(S1):84-5.

16. Lloyd M, MacDonald M, Lord C. Motor skills of toddlers with autism spectrum disorders. Autism (2013) 17(2):133-46. doi:10.1177/1362361311402230

17. Pitchford EA, MacDonald M, Hauck JL, Ulrich DA. Activity participation among youth with autism spectrum disorders. Res Q Exerc Sport (2013) 84(S1):91-2.

18. Staples KL, MacDonald M, Zimmer C. Assessment of motor behavior among children and adolescents with autism spectrum disorder. Int Rev Res Dev Dis (2012) 42:179-214. doi:10.1016/B978-0-12-394284-5.00007-3

19. Zimmer C, Staples K. Motor Performance of Children with Autism Spectrum Disorders. Regina: University of Regina Graduate Students' Association (2011).

20. Staples KL, Reid G. Fundamental movement skills and autism spectrum disorders. J Autism Dev Disord (2010) 40(2):209-17. doi:10.1007/ s10803-009-0854-9 
21. Reilly J. Can we modulate physical activity in children? Int J Obes (2011) 35(10):1266-9. doi:10.1038/ijo.2011.62

22. Welk GJ, Corbin CB, Dale D. Measurement issues in the assessment of physical activity in children. Res Q Exerc Sport (2000) 71(Suppl 2):59-73. doi:10.1080/ 02701367.2000.11082788

23. Hauck JL, Ulrich DA. Acute effects of a therapeutic mobility device on physical activity and heart rate in children with Down syndrome. Res Q Exerc Sport (2015) 86(3):260-6. doi:10.1080/02701367.2015.1046980

24. Hauck JL. Strategies for adapting accelerometer wear for youth with disabilities. Suppl Res Q Exer Sport (2011) 82(S1):71.

25. Jeong I. Measuring Physical Activity in Youth with Down Syndrome and Autism Spectrum Disorders: Identifying Data-Based Measurement Conditions [Dissertation]. Ann Arbor: University of Michigan (2012).

26. Trost SG, Pate RR, Freedson PS, Sallis JF, Taylor WC. Using objective physical activity measures with youth: how many days of monitoring are needed? Med SciSportsExerc (2000) 32(2):426-31. doi:10.1097/00005768-200002000-00025

27. SoYeun K, JoonKoo Y. Determining daily physical activity levels of youth with developmental disabilities: days of monitoring required? Adapted Phys Activity $Q$ (2009) 26(3):220-35. doi:10.1123/apaq.26.3.220

28. Hauck JL, Jeong I, Esposito P, MacDonald M, Hornyak JE, Argento A, et al. Benefits of learning to ride a two-wheeled bicycle for adolescents with developmental disabilities. Palaestra (2017) 31(3).

29. Reynolds JL, Pitchford EA, Hauck JL, Ketcheson LR, Ulrich DA. Outcomes of home-support consultation on the maintenance of bicycle-riding skills for youth with autism spectrum disorder. JEduc Psychol Consult (2016) 26(2):166-85. doi:10.1080/10474412.2015.1067147
30. MacDonald M, Esposito P, Hauck J, Jeong I, Hornyak J, Argento A, et al. Bicycle training for youth with Down syndrome and autism spectrum disorders. Focus Autism Other Dev Disabl (2012) 27(1):12-21. doi:10.1177/1088357611428333

31. Puyau MR, Adolph AL, Vohra FA, Zakeri I, Butte NF. Prediction of activity energy expenditure using accelerometers in children. Med Sci Sports Exerc (2004) 36(9):1625-31. doi:10.1249/01.MSS.0000139898.30804.60

32. Puyau MR, Adolph AL, Vohra FA, Butte NF. Validation and calibration of physical activity monitors in children. Obes Res (2002) 10(3):150-7. doi:10.1038/oby.2002.24

33. Pfeiffer KA, Mciver KL, Dowda M, Almeida M, Pate RR. Validation and calibration of the Actical accelerometer in preschool children. Med Sci Sports Exerc (2006) 38(1):152-7. doi:10.1249/01.mss.0000183219.44127.e7

34. Ketcheson LR. Motor Skills and Level of Physical Activity in Young Children with Autism Spectrum Disorder [Dissertation]. Ann Arbor: University of Michigan (2014).

Conflict of Interest Statement: The authors declare that the research was conducted in the absence of any commercial or financial relationships that could be construed as a potential conflict of interest.

Copyright (c) 2016 Hauck, Ketcheson and Ulrich. This is an open-access article distributed under the terms of the Creative Commons Attribution License (CC BY). The use, distribution or reproduction in other forums is permitted, provided the original author(s) or licensor are credited and that the original publication in this journal is cited, in accordance with accepted academic practice. No use, distribution or reproduction is permitted which does not comply with these terms. 